\title{
Self-Assembled, Iron-Functionalized Polyoxovanadate Alkoxide Clusters
}

Feng Li, Lauren E. VanGelder, William W. Brennessel and Ellen M. Matson*

Department of Chemistry, University of Rochester, Rochester, New York 14627, United States

\section{Supporting Information Table of Contents:}

Experimental

Table S1. Experimental Conditions for Reaction Profile Determination

Figure S1. Reaction Profile and ESI-MS of $5 \mathrm{VO}\left(\mathrm{O}^{t} \mathrm{Bu}\right)_{3}+\mathrm{FeCl}_{2}$ in $\mathrm{MeOH}$ at room temperature

Figure S2. Reaction Profile and ESI-MS of $\mathrm{VO}\left(\mathrm{O}^{t} \mathrm{Bu}\right)_{3}+\mathrm{FeCl}_{2}$ in $\mathrm{MeOH}$ at room temperature.....

Figure S3. Reaction Profile and ESI-MS of $\mathrm{VO}\left(\mathrm{O}^{\mathrm{t}} \mathrm{Bu}\right)_{3}+\mathrm{FeCl}_{2}$ in $\mathrm{MeOH}$ at $60{ }^{\circ} \mathrm{C}$........ .S4

Figure S4. Reaction Profile and ESI-MS of $3 \mathrm{VO}\left(\mathrm{O}^{t} \mathrm{Bu}\right)_{3}+\mathrm{FeCl}_{2}$ in $\mathrm{MeOH}$ at $60^{\circ} \mathrm{C}$. .S5

Figure S5. Reaction Profile and ESI-MS of $5 \mathrm{VO}\left(\mathrm{O}^{\mathrm{t}} \mathrm{Bu}\right)_{3}+\mathrm{FeCl}_{2}$ in $\mathrm{MeOH}$ at $60{ }^{\circ} \mathrm{C}$ .S5

Figure S6. Reaction Profile and ESI-MS of $10 \mathrm{VO}\left(\mathrm{O}^{t} \mathrm{Bu}\right)_{3}+\mathrm{FeCl}_{2}$ in $\mathrm{MeOH}$ at $60{ }^{\circ} \mathrm{C}$ .S5

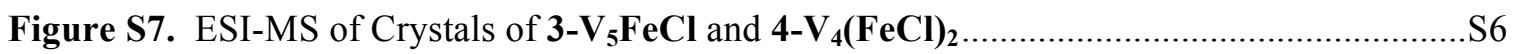

Figure S8. Reaction Profile and ESI-MS of $3 \mathrm{VO}\left(\mathrm{O}^{t} \mathrm{Bu}\right)_{3}+\mathrm{FeBr}_{2}$ in $\mathrm{MeOH}$ at $60^{\circ} \mathrm{C}$...............S6

Figure S9. Reaction Profile and ESI-MS of $3 \mathrm{VO}(\mathrm{OMe})_{3}+\mathrm{FeCl}_{2}$ in Toluene at $85^{\circ} \mathrm{C}$.............S6

Figure S10. Reaction Profile and ESI-MS of $3 \mathrm{VO}(\mathrm{OMe})_{3}+\mathrm{FeBr}_{2}$ in Toluene at $85^{\circ} \mathrm{C} \ldots \ldots \ldots . . . . \mathrm{S} 7$

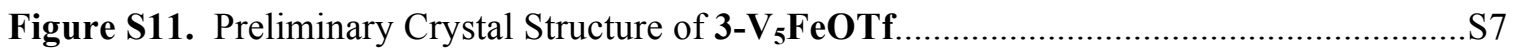

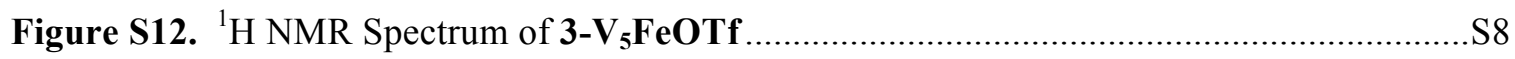

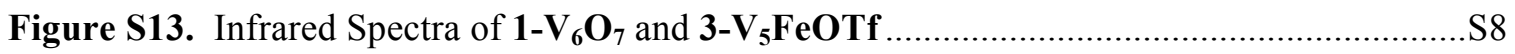

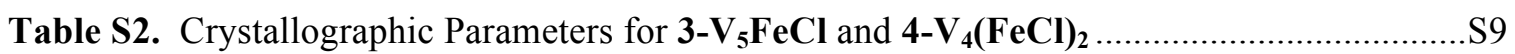

References. 


\section{Experimental.}

General Considerations. All manipulations were carried out in the absence of water and dioxygen using standard Schlenk techniques, or in an UniLab MBraun inert atmosphere drybox under a dinitrogen atmosphere except where specified otherwise. All glassware was oven dried for a minimum of $8 \mathrm{~h}$ and cooled in an evacuated antechamber prior to use in the drybox. Unless otherwise noted, solvents were dried and deoxygenated on a Glass Contour System (Pure Process Technology, LLC) and stored over activated $3 \AA$ molecular sieves purchased from Fisher Scientific prior to use. Methanol (anhydrous, 99.8\%) was purchased from Sigma Aldrich and used as received. $\mathrm{VO}(\mathrm{OCH})_{3},{ }^{1}$ and $\mathrm{VO}\left(\mathrm{O}^{t} \mathrm{Bu}\right)_{3}{ }^{2}$ were prepared according to published procedures. $\mathrm{FeCl}_{2}$ (anhydrous, 98\%) and $\mathrm{FeBr}_{2}$ (anhydrous, 98\%) were purchased from Strem and used without further purification. Celite 545 (J. T. Baker) was dried in a Schlenk flask for $24 \mathrm{~h}$ under vacuum while heating to at least $150{ }^{\circ} \mathrm{C}$ prior to use in the glovebox. Elemental analyses were performed on a PerkinElmer 2400 Series II Analyzer, at the CENTC Elemental Analysis Facility, University of Rochester.

The ${ }^{1} \mathrm{H}$ NMR spectra was recorded at 500 and $400 \mathrm{MHz}$ on Bruker DPX-500 and Bruker DPX-400 MHz spectrometers locked on the signal of deuterated solvents. All chemical shifts were reported relative to the peak of residual $\mathrm{H}$ signal in deuterated solvents. $\mathrm{CDCl}_{3}$ and $\mathrm{C}_{6} \mathrm{D}_{6}$ were purchased from Cambridge Isotope Laboratories, degassed by three freeze-pump-thaw cycles, and stored over fully activated $3 \AA$ molecular sieves. Infrared (FT-IR, ATR) spectra of complexes were recorded on a Shimadzu IRAffinity-1 Fourier Transform Infrared Spectrophotometer and are reported in wavenumbers $\left(\mathrm{cm}^{-1}\right)$. Electronic absorption measurements were recorded at room temperature in anhydrous acetonitrile and methanol in a sealed $1 \mathrm{~cm}$ quartz cuvette with an Agilent Cary $60 \mathrm{UV}-\mathrm{V}$ is spectrophotometer. Mass spectrometry analyses were performed on a Thermo LTQ Velos LC/MS equipped with an electrospray probe operating in positive ion mode (ESI+) with an ion trap mass analyzer. Direct injection analysis was employed in all cases with a sample solution in methanol. Single crystals of $\mathbf{3}-\mathbf{V}_{\mathbf{5}} \mathbf{F e C l}$ and 3$\mathbf{V}_{5} \mathbf{F e O T f}$ were mounted on the tip of a thin glass optical fiber (goniometer head) and mounted on a Bruker SMART APEX II CCD platform diffractometer for a data collection at 100.0(5) K. The structure was solved using SHELXT-2014/5 ${ }^{3}$ and refined using SHELXL-2014/7 ${ }^{4}$.

Cyclic Voltammograms were recorded with a $\mathrm{CH}$ Instruments Inc. 410c time-resolved electrochemical quartz crystal microbalance. All measurements were performed in a three electrode system cell configuration that consisted of a glassy-carbon $(\varnothing=3.0 \mathrm{~mm})$ as working electrode, a Pt wire as the counter electrode, and an $\mathrm{Ag} / \mathrm{AgCl}$ wire as the reference electrode. All electrochemical measurements were performed at room temperature in a $\mathrm{N}_{2}$-filled glovebox. Dry dichloromethane that contained $0.1 \mathrm{M}^{n} \mathrm{Bu}_{4} \mathrm{NPF}_{6}$ was used as the electrolyte solution. All redox events were referenced against ferrocenium/ferrocene $\left(\mathrm{Fc}^{+} / \mathrm{Fc}\right)$ redox couple.

Synthesis of $(\mathrm{VO})_{5} \mathrm{O}\left(\mathrm{OCH}_{3}\right)_{12} \mathrm{Fe}\left(\mathrm{SO}_{3} \mathrm{CF}_{3}\right)\left(3-\mathrm{V}_{5} \mathbf{F e O T f}\right)$. In the glovebox, $\mathrm{VO}\left(\mathrm{OCH}_{3}\right)_{3}(0.360 \mathrm{~g}$, $2.25 \mathrm{mmol}), \mathrm{FeBr}_{2}(0.162 \mathrm{~g}, 0.75 \mathrm{mmol})$, and toluene $(18 \mathrm{~mL})$ were placed in four $48 \mathrm{~mL}$ heavy wall pressure vessels, which were then taken out of the glovebox and heated in an oil bath at 85 ${ }^{\circ} \mathrm{C}$ for 110 hours with stirring. Dark brown-green suspensions were eventually obtained, united and filtered over a bed of Celite $(1.0 \mathrm{~cm})$ on a medium porosity glass frit. Solvent from the resulting dark green solution was removed under reduced pressure. To the solid residue was added $\mathrm{AgSO}_{3} \mathrm{CF}_{3}$ (AgOTf, $0.526 \mathrm{~g}, 2.05 \mathrm{mmol}$ ) and $\mathrm{MeCN}(120 \mathrm{~mL}$ ). The reaction mixture was stirred vigorously for $1.5 \mathrm{hrs}$, during which a yellow precipitate formed. After the removal of the precipitate by filtration, and removal of solvent under reduced pressure, $\mathrm{Et}_{2} \mathrm{O}(20 \mathrm{~mL} \times 2)$ was added to extract the product. The solution was then concentrated under reduced pressure into two thirds of the original volume, and left for slow evaporation. After two days, a large amount of dark green crystalline material was formed on the wall of the vial. Yield: $1.382 \mathrm{~g}(76.8 \%$ based 
on $\left.\mathrm{VO}(\mathrm{OMe})_{3}\right)$. Single crystals suitable for X-ray crystallography were selected from the crystalline materials. ${ }^{1} \mathrm{H}$ NMR $\left(400 \mathrm{MHz}, \mathrm{CDCl}_{3}\right): \delta=26.37(902 \mathrm{~Hz}, 24 \mathrm{H}), 11.71(1273 \mathrm{~Hz}$, 12H). FT-IR (ATR, $\mathrm{cm}^{-1}$ ) 2922, 2816, 1443, 1234, 1173, 1012, 974, 876, 804, 632. UV-Vis $\left(\mathrm{CH}_{3} \mathrm{OH}\right)\left[\varepsilon\left(\mathrm{M}^{-1} \mathrm{~cm}^{-1}\right)\right]: 204 \mathrm{~nm}\left(3.99 \times 10^{4}\right), 296 \mathrm{~nm}\left(1.38 \times 10^{4}\right), 378 \mathrm{~nm}\left(8.21 \times 10^{3}\right), 982 \mathrm{~nm}$ $\left(6.96 \times 10^{2}\right) ; \mathrm{UV}-\mathrm{Vis}\left(\mathrm{CH}_{3} \mathrm{CN}\right)\left[\varepsilon\left(\mathrm{M}^{-1} \mathrm{~cm}^{-1}\right)\right]: 256 \mathrm{~nm}\left(1.94 \times 10^{4}\right), 308 \mathrm{~nm}\left(1.47 \times 10^{4}\right), 378$ $\mathrm{nm}\left(1.07 \times 10^{4}\right), 982 \mathrm{~nm}\left(7.54 \times 10^{2}\right)$. Elemental analysis Calcd $(\%)$ for $\mathrm{C}_{13} \mathrm{H}_{36} \mathrm{O}_{21} \mathrm{~F}_{3} \mathrm{SV}_{5} \mathrm{Fe}(\mathrm{MW}$ $=928.02 \mathrm{~g} / \mathrm{mol}): \mathrm{C}, 16.82 ; \mathrm{H}, 3.91 ; \mathrm{N}, 0.00$. Found: $\mathrm{C}, 16.61 ; \mathrm{H}, 3.51 ; \mathrm{N},-0.01$.

General Description of Reaction Conditions for Profile Experiments. In the glovebox, a $15 \mathrm{~mL}$ heavy wall pressure vessel was charged with $\mathrm{VO}\left(\mathrm{OR}_{3}\right)_{3}\left(\mathrm{R}={ }^{\mathrm{t}} \mathrm{Bu}, \mathrm{Me}\right), \mathrm{FeX}_{2}(\mathrm{X}=\mathrm{Cl}, \mathrm{Br})$, and solvent. This pressure vessel was then sealed and removed from the glovebox and heated in an oil bath at a given temperature with stirring. To collect each time point in the reaction profile, the pressure vessel was removed from the oil bath and briefly returned to the glove box so that an aliquot could be removed for ESI-MS analysis. Once the aliquot was obtained, the reaction mixture was returned to the oil bath outside the glove box in manner analogous to that described earlier. This procedure was adapted for the remaining reaction profile experiments with appropriate conditions and molecular equivalents.

Table S1. Experimental Conditions for Reaction Profile Determination

\begin{tabular}{|c|c|c|c|c|c|}
\hline $\begin{array}{c}\text { General } \\
\text { Reaction } \\
\text { Conditions }\end{array}$ & $\begin{array}{l}\text { Amount of } \\
\operatorname{VO}(\mathrm{OR})_{3}\end{array}$ & $\begin{array}{l}\text { Amount of } \\
\mathrm{FeX}_{2}\end{array}$ & $\begin{array}{c}\text { Volume of } \\
\text { Solvent }(\mathrm{mL})\end{array}$ & $\begin{array}{c}\text { Temperature } \\
\left({ }^{\circ} \mathrm{C}\right)\end{array}$ & Figure \\
\hline $\begin{array}{c}5 \mathrm{VO}\left(\mathrm{O}^{t} \mathrm{Bu}\right)_{3}+ \\
\mathrm{FeCl}_{2} \text { in } \mathrm{MeOH}\end{array}$ & $\begin{array}{c}258.6 \mathrm{mg} \\
(0.907 \mathrm{mmol}) \\
\mathrm{R}={ }^{\mathrm{t}} \mathrm{Bu}\end{array}$ & $\begin{array}{c}22.8 \mathrm{mg} \\
(0.180 \mathrm{mmol}) \\
\mathrm{X}=\mathrm{Cl}\end{array}$ & $8 \mathrm{~mL}$ & 25 (Room Temp) & S1 \\
\hline $\begin{array}{c}\mathrm{VO}\left(\mathrm{O}^{t} \mathrm{Bu}\right)_{3}+ \\
\mathrm{FeCl}_{2} \text { in } \mathrm{MeOH}\end{array}$ & $\begin{array}{c}258.8 \mathrm{mg} \\
(0.908 \mathrm{mmol}) \\
\mathrm{R}={ }^{\mathrm{t}} \mathrm{Bu}\end{array}$ & $\begin{array}{c}114 \mathrm{mg} \\
(0.899 \mathrm{mmol}) \\
\mathrm{X}=\mathrm{Cl}\end{array}$ & $8 \mathrm{~mL}$ & 25 (Room Temp) & S2 \\
\hline $\begin{array}{c}\mathrm{VO}\left(\mathrm{O}^{\mathrm{t}} \mathrm{Bu}\right)_{3}+ \\
\mathrm{FeCl}_{2} \text { in } \mathrm{MeOH}\end{array}$ & $\begin{array}{c}258.4 \mathrm{mg} \\
(0.907 \mathrm{mmol}) \\
\mathrm{R}={ }^{\mathrm{t}} \mathrm{Bu}\end{array}$ & $\begin{array}{c}114 \mathrm{mg} \\
(0.899 \mathrm{mmol}) \\
\mathrm{X}=\mathrm{Cl}\end{array}$ & $8 \mathrm{~mL}$ & 60 & S3 \\
\hline $\begin{array}{c}3 \mathrm{VO}\left(\mathrm{O}^{\mathrm{t}} \mathrm{Bu}\right)_{3}+ \\
\mathrm{FeCl}_{2} \text { in } \mathrm{MeOH}\end{array}$ & $\begin{array}{c}259.4 \mathrm{mg} \\
(0.910 \mathrm{mmol}) \\
\mathrm{R}={ }^{\mathrm{t}} \mathrm{Bu}\end{array}$ & $\begin{array}{c}38 \mathrm{mg} \\
(0.300 \mathrm{mmol}) \\
\mathrm{X}=\mathrm{Cl}\end{array}$ & $8 \mathrm{~mL}$ & 60 & S4 \\
\hline $\begin{array}{c}5 \mathrm{VO}\left(\mathrm{O}^{\mathrm{t}} \mathrm{Bu}\right)_{3}+ \\
\mathrm{FeCl}_{2} \text { in } \mathrm{MeOH}\end{array}$ & $\begin{array}{c}259.2 \mathrm{mg} \\
(0.909 \mathrm{mmol}) \\
\mathrm{R}={ }^{\mathrm{t}} \mathrm{Bu}\end{array}$ & $\begin{array}{c}22.8 \mathrm{mg} \\
(0.180 \mathrm{mmol}) \\
\mathrm{X}=\mathrm{Cl}\end{array}$ & $8 \mathrm{~mL}$ & 60 & S5 \\
\hline $\begin{array}{l}10 \mathrm{VO}\left(\mathrm{O}^{t} \mathrm{Bu}\right)_{3}+ \\
\mathrm{FeCl}_{2} \text { in } \mathrm{MeOH}\end{array}$ & $\begin{array}{c}258.7 \mathrm{mg} \\
(0.908 \mathrm{mmol}) \\
\mathrm{R}={ }^{\mathrm{t}} \mathrm{Bu} \\
\end{array}$ & $\begin{array}{c}11.4 \mathrm{mg} \\
(0.090 \mathrm{mmol}) \\
\mathrm{X}=\mathrm{Cl} \\
\end{array}$ & $8 \mathrm{~mL}$ & 60 & S6 \\
\hline $\begin{array}{c}3 \mathrm{VO}\left(\mathrm{O}^{t} \mathrm{Bu}\right)_{3}+ \\
\mathrm{FeBr}_{2} \text { in } \mathrm{MeOH}\end{array}$ & $\begin{array}{c}129.1 \mathrm{mg} \\
(0.453 \mathrm{mmol}) \\
\mathrm{R}={ }^{\mathrm{t}} \mathrm{Bu}\end{array}$ & $\begin{array}{c}33 \mathrm{mg} \\
(0.153 \mathrm{mmol}) \\
\mathrm{X}=\mathrm{Br}\end{array}$ & $4 \mathrm{~mL}$ & 60 & S8 \\
\hline $\begin{array}{c}3 \mathrm{VO}(\mathrm{OMe})_{3}+ \\
\mathrm{FeCl}_{2} \text { in Toluene }\end{array}$ & $\begin{array}{c}203.0 \mathrm{mg} \\
(1.269 \mathrm{mmol}) \\
\mathrm{R}=\mathrm{Me}\end{array}$ & $\begin{array}{c}52.7 \mathrm{mg} \\
(0.416 \mathrm{mmol}) \\
\mathrm{X}=\mathrm{Cl}\end{array}$ & $10 \mathrm{~mL}$ & 85 & S9 \\
\hline $\begin{array}{c}3 \mathrm{VO}(\mathrm{OMe})_{3}+ \\
\mathrm{FeBr}_{2} \text { in Toluene }\end{array}$ & $\begin{array}{c}201.2 \mathrm{mg} \\
(1.258 \mathrm{mmol}) \\
\mathrm{R}=\mathrm{Me}\end{array}$ & $\begin{array}{c}90.6 \mathrm{mg} \\
(0.420 \mathrm{mmol}) \\
\mathrm{X}=\mathrm{Br}\end{array}$ & $10 \mathrm{~mL}$ & 85 & S10 \\
\hline
\end{tabular}



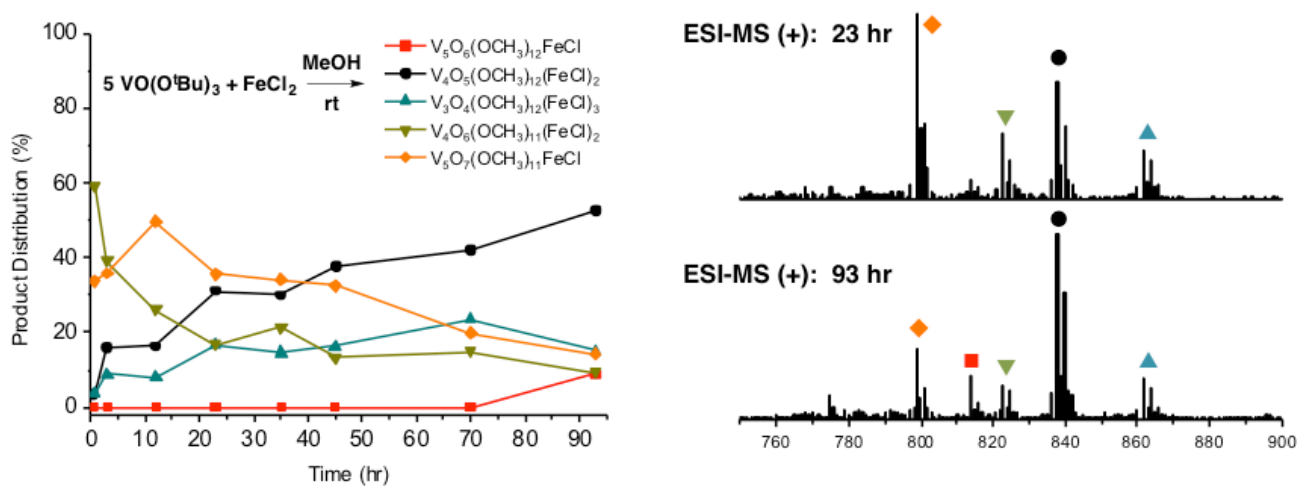

Figure S1. Reaction Profile and ESI-MS of $5 \mathrm{VO}\left(\mathrm{O}^{t} \mathrm{Bu}\right)_{3}+\mathrm{FeCl}_{2}$ in $\mathrm{MeOH}$ at room temperature
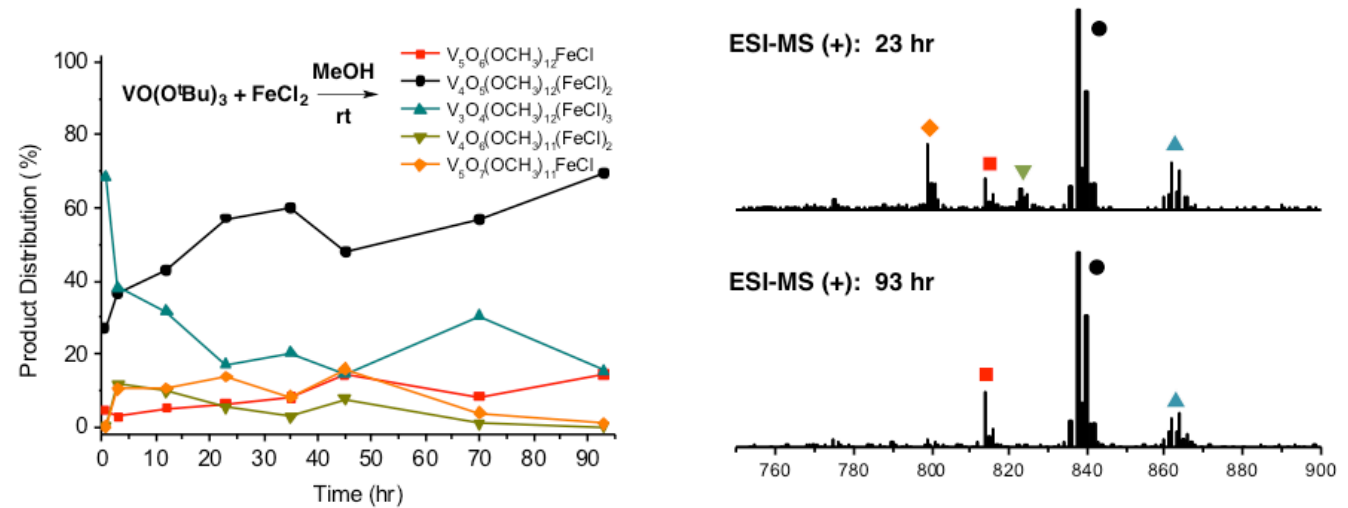

Figure S2. Reaction Profile and ESI-MS of $\mathrm{VO}\left(\mathrm{O}^{t} \mathrm{Bu}\right)_{3}+\mathrm{FeCl}_{2}$ in $\mathrm{MeOH}$ at room temperature
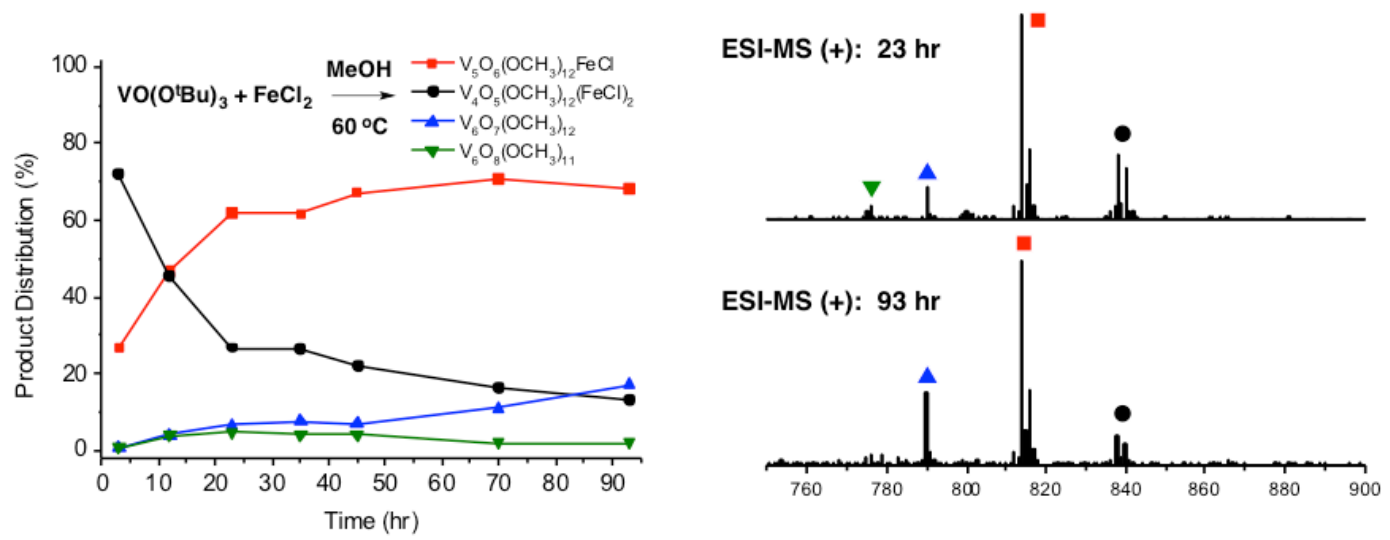

Figure S3. Reaction Profile and ESI-MS of $\mathrm{VO}\left(\mathrm{O}^{t} \mathrm{Bu}\right)_{3}+\mathrm{FeCl}_{2}$ in $\mathrm{MeOH}$ at $60^{\circ} \mathrm{C}$. 

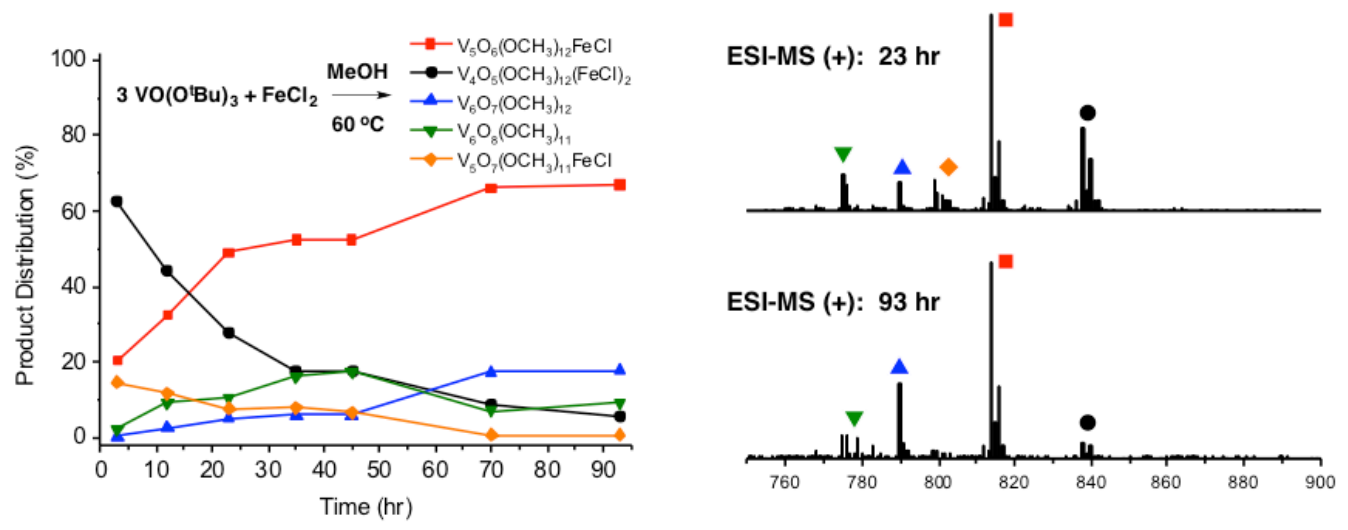

Figure S4. Reaction Profile and ESI-MS of $3 \mathrm{VO}\left(\mathrm{O}^{t} \mathrm{Bu}\right)_{3}+\mathrm{FeCl}_{2}$ in $\mathrm{MeOH}$ at $60^{\circ} \mathrm{C}$.
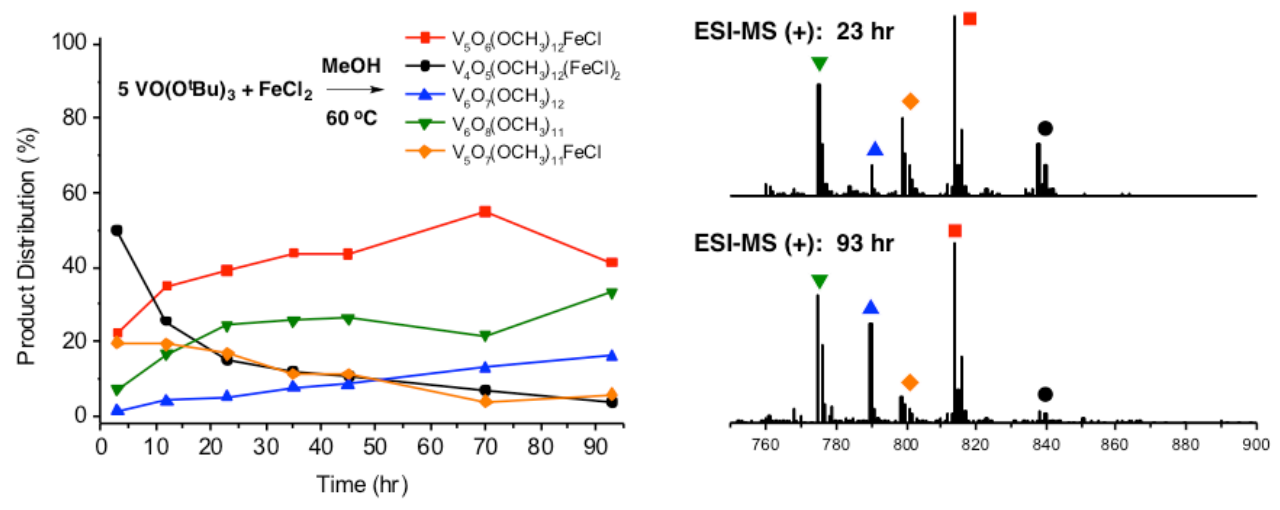

Figure S5. Reaction Profile and ESI-MS of $5 \mathrm{VO}\left(\mathrm{O}^{\mathrm{t}} \mathrm{Bu}\right)_{3}+\mathrm{FeCl}_{2}$ in $\mathrm{MeOH}$ at $60{ }^{\circ} \mathrm{C}$
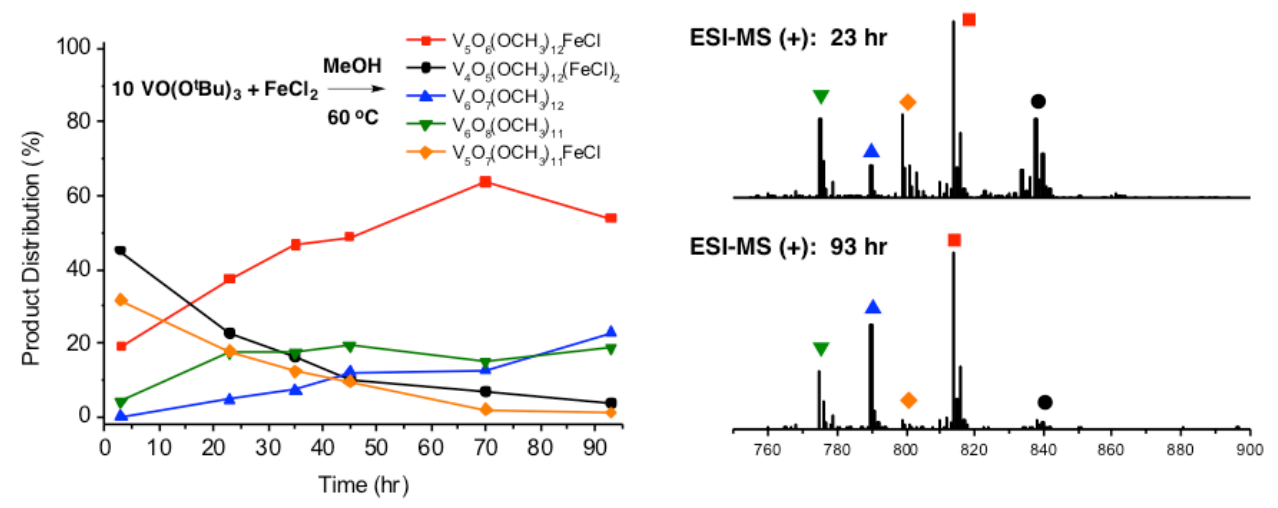

Figure S6. Reaction Profile and ESI-MS of $10 \mathrm{VO}\left(\mathrm{O}^{t} \mathrm{Bu}\right)_{3}+\mathrm{FeCl}_{2}$ in $\mathrm{MeOH}$ at $60^{\circ} \mathrm{C}$. 


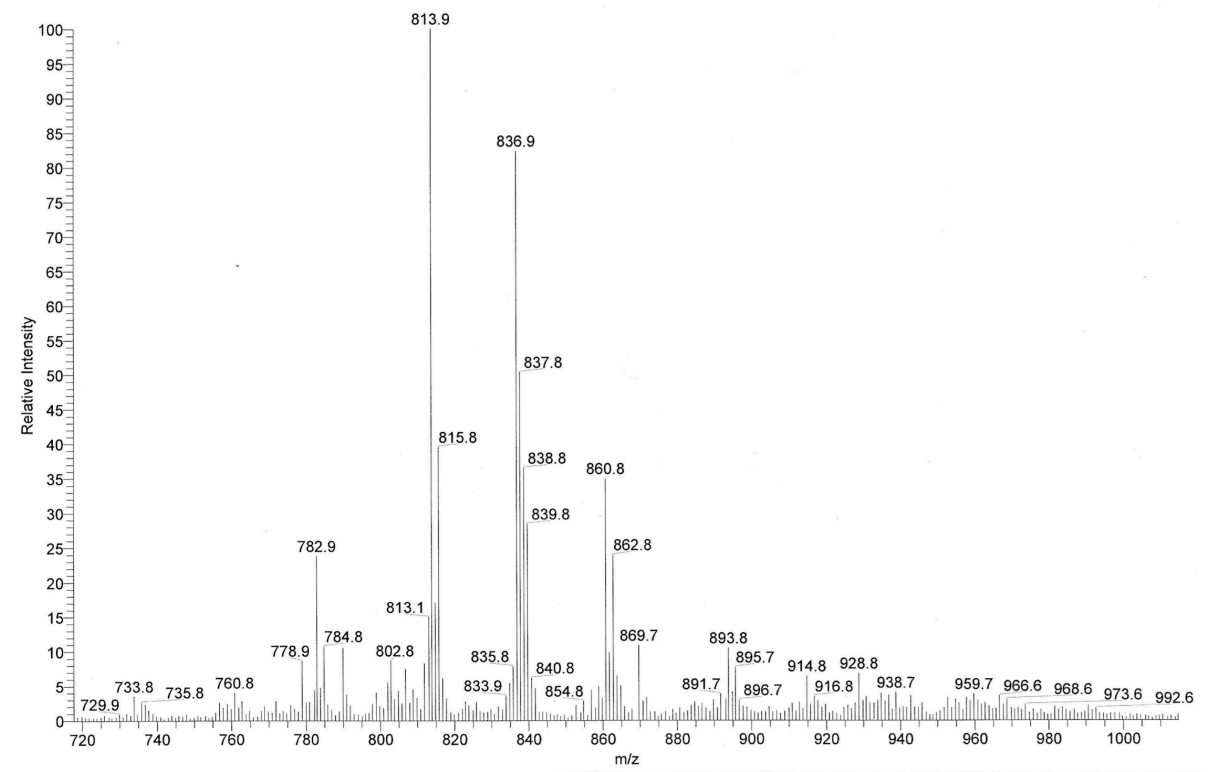

Figure S7. ESI-MS of Crystals of $3-\mathrm{V}_{5} \mathbf{F e C l}$ and $4-\mathrm{V}_{4}(\mathbf{F e C l})_{2}$
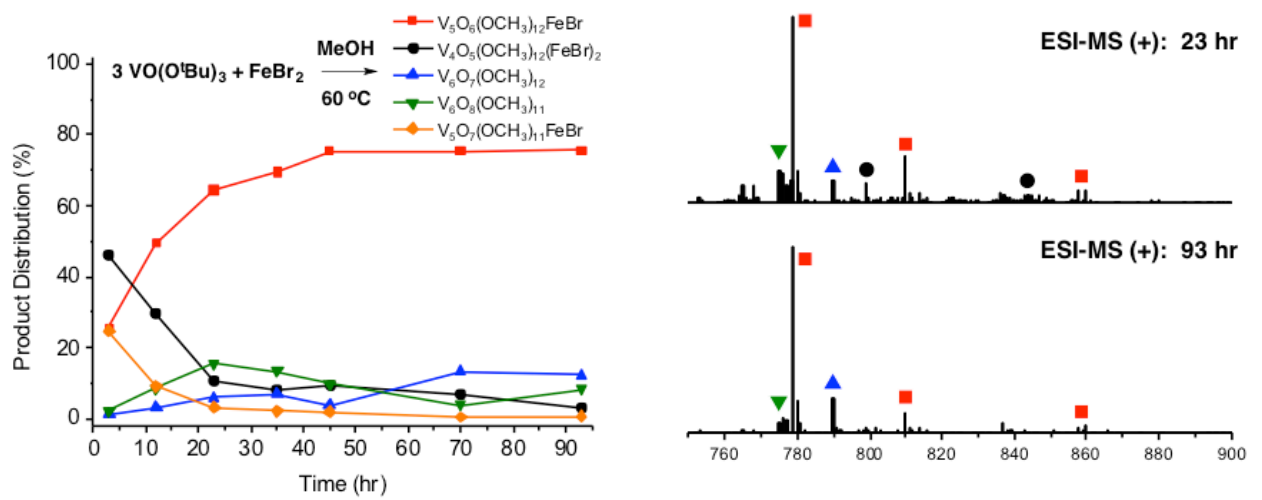

Figure S8. Reaction Profile and ESI-MS of $3 \mathrm{VO}\left(\mathrm{O}^{t} \mathrm{Bu}\right)_{3}+\mathrm{FeBr}_{2}$ in $\mathrm{MeOH}$ at $60^{\circ} \mathrm{C}^{*}$
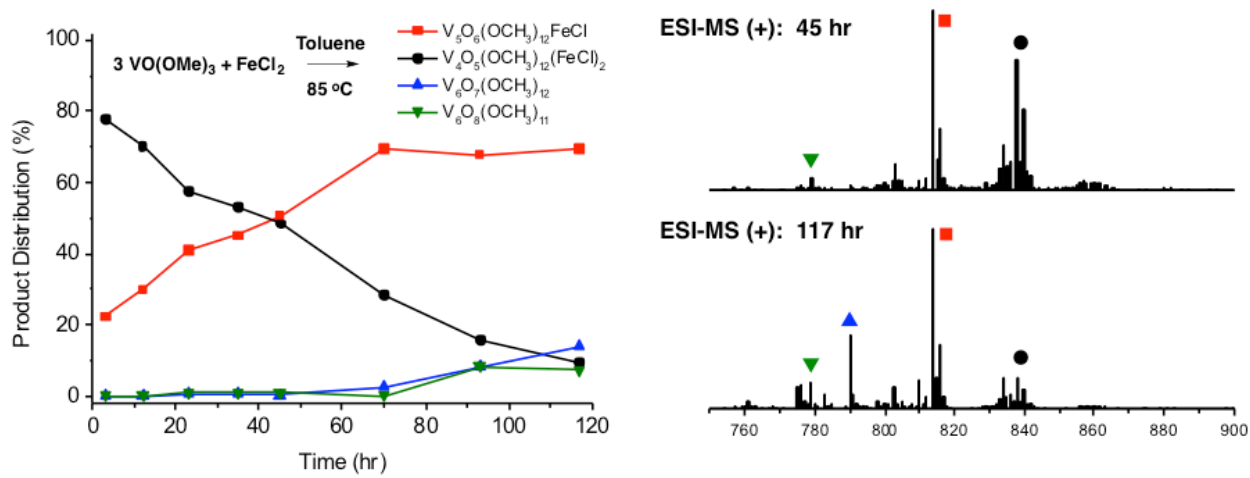

Figure S9. Reaction Profile and ESI-MS of $3 \mathrm{VO}(\mathrm{OMe})_{3}+\mathrm{FeCl}_{2}$ in Toluene at $85^{\circ} \mathrm{C}$. 

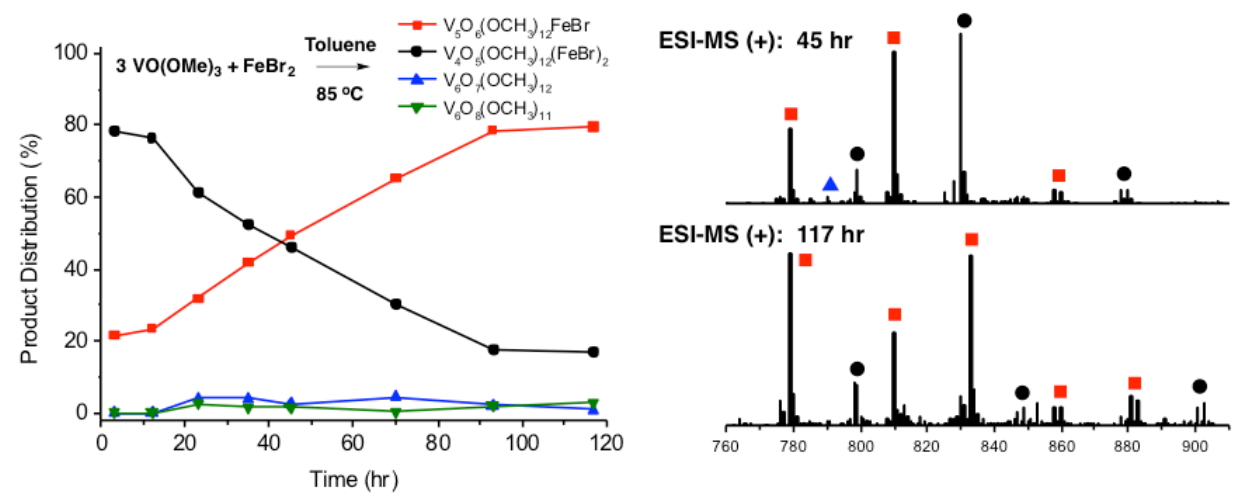

Figure S10. Reaction Profile and ESI-MS of $3 \mathrm{VO}(\mathrm{OMe})_{3}+\mathrm{FeBr}_{2}$ in Toluene at $85^{\circ} \mathrm{C}^{*}$

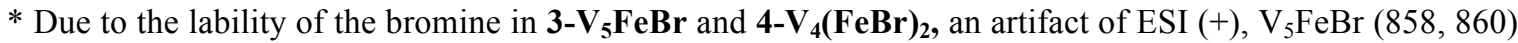
in methanol was observed as $\mathrm{V}_{5} \mathrm{Fe}(779), \mathrm{V}_{5} \mathrm{FeOMe}(810)$, and peaks with $\mathrm{Na}+$ attached. $\mathrm{V}_{4}(\mathrm{FeBr})_{2}(926$, 928, 930) in methanol was observed as $\mathrm{V}_{4} \mathrm{Fe}_{2}$ (768), $\mathrm{V}_{4} \mathrm{Fe}(\mathrm{FeOMe})(799), \mathrm{V}_{4}(\mathrm{FeOMe})_{2}$ (830), $\mathrm{V}_{4}(\mathrm{FeOMe})(\mathrm{FeBr})(878,880)$ and peaks with $\mathrm{Na}^{+}$attached.

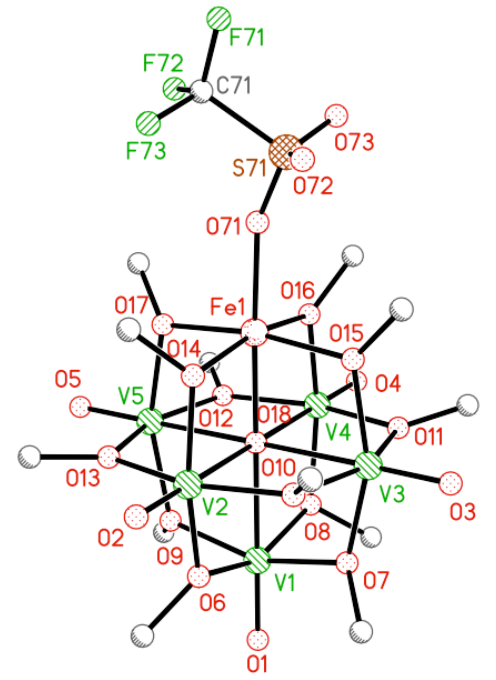

Figure S11. Preliminary molecular structure of $3-V_{5}$ FeOTf. Triflate anions were not able to be modeled appropriately, resulting in a large $\mathrm{R}$ factor for this structure. The molecule crystallized

in the space group P 21/c. Three independent clusters were modeled in a single unit cell (parameters below), adding to the high level of disorder. No disorder was noted in the cluster core itself, confirming the assembly of the heterometallic cluster $3-V_{5} \mathbf{F e O T f}$.

$$
\begin{array}{ll}
\mathrm{a}=22.725 & \alpha=90 \\
\mathrm{~b}=15.115 & \beta=90 \\
\mathrm{c}=28.685 & \gamma=90
\end{array}
$$




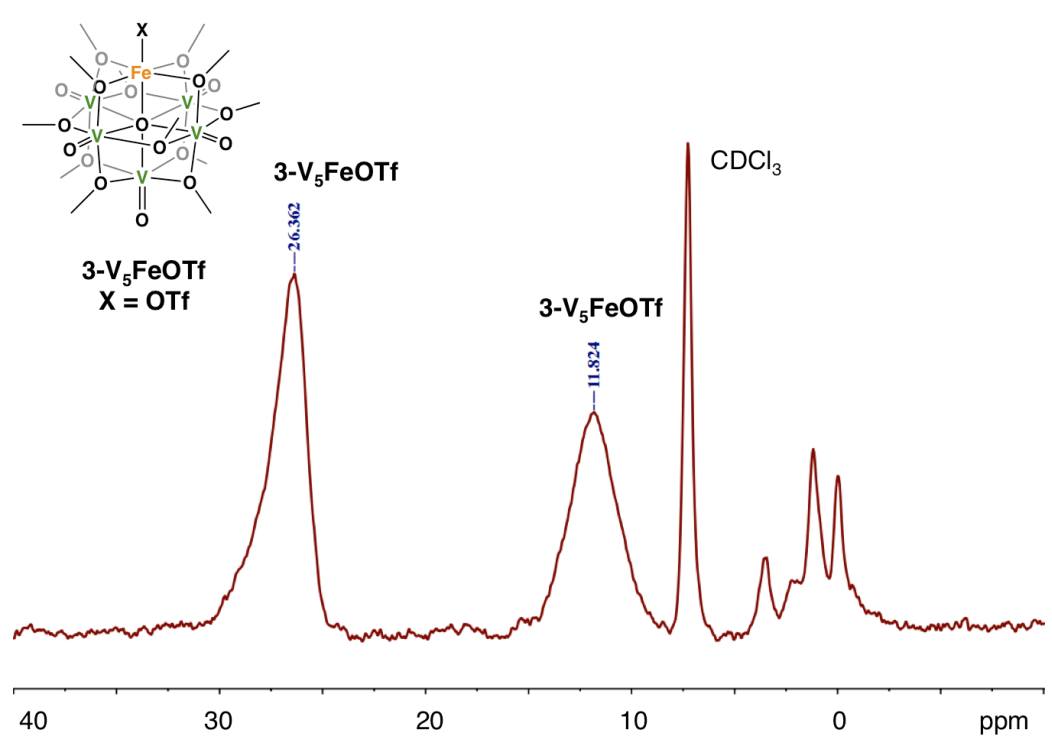

Figure S12. . ${ }^{1} \mathrm{H}$ NMR Spectrum $\left(\mathrm{CDCl}_{3}, 21^{\circ} \mathrm{C}\right)$ of $\mathbf{3 - V _ { 5 }} \mathbf{F e O T f}$.

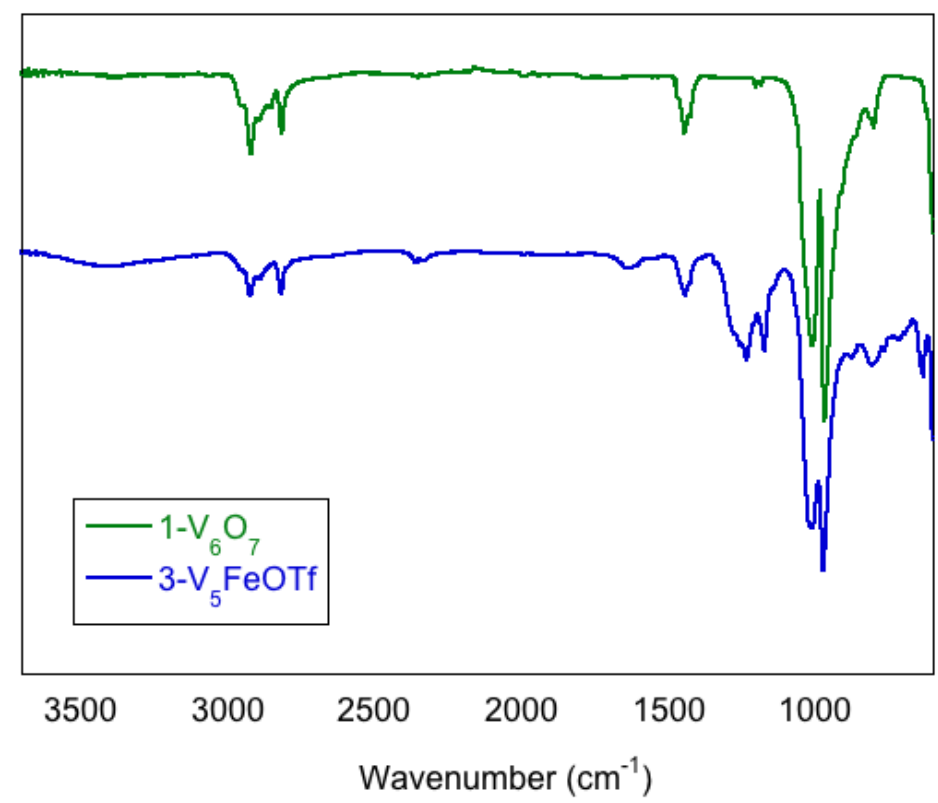

Figure S13. Infrared Spectra of $1-V_{6} O_{7}$ and $3-V_{5}$ FeOTf

Table S2. Crystallographic parameters for the disordered structure of $3-\mathbf{V}_{5} \mathbf{F e C l}$ and $4-\mathbf{V}_{4}(\mathbf{F e C l})_{2}$. 


\begin{tabular}{|c|c|c|}
\hline & \multicolumn{2}{|c|}{$3-\mathrm{V}_{5} \mathrm{FeCl} / 4-\mathrm{V}_{4}(\mathrm{FeCl})_{2}$} \\
\hline Empirical formula & \multicolumn{2}{|c|}{$\mathrm{C}_{12} \mathrm{H}_{36} \mathrm{Cl}_{1.74} \mathrm{Fe}_{1.74} \mathrm{O}_{17.26} \mathrm{~V}_{4.26}$} \\
\hline Formula weight & \multicolumn{2}{|l|}{832.43} \\
\hline Temperature & \multicolumn{2}{|l|}{$100.0(5)$} \\
\hline Wavelength & \multicolumn{2}{|l|}{0.71073} \\
\hline Crystal system & \multicolumn{2}{|l|}{ Monoclinic } \\
\hline Space group & \multicolumn{2}{|l|}{$\mathrm{P} 21 / \mathrm{n}$} \\
\hline Unit cell dimensions & $\begin{array}{l}\mathrm{a}=9.6791(4) \\
\mathrm{b}=9.7647(4) \\
\mathrm{c}=15.2953(6)\end{array}$ & $\begin{array}{l}\alpha=90 \\
\beta=90.1320(9) \\
\gamma=90\end{array}$ \\
\hline Volume & \multicolumn{2}{|l|}{$1445.61(10)$} \\
\hline $\mathrm{Z}$ & \multicolumn{2}{|l|}{2} \\
\hline Reflections collected & \multicolumn{2}{|l|}{6961} \\
\hline Independent reflections & \multicolumn{2}{|l|}{4094} \\
\hline Goodness-of-fit on F2 & \multicolumn{2}{|l|}{1.060} \\
\hline $\begin{array}{l}\text { Final } R \text { indices } \\
{[\mathrm{I}>2 \operatorname{sigma}(\mathrm{I})]}\end{array}$ & \multicolumn{2}{|l|}{0.0467} \\
\hline
\end{tabular}

\section{References.}

1. Daniel, C.; Hartl, H. J. Am. Chem. Soc. 2009, 131, 5101-5114.

2. Pak, C.; Bell, A. T.; Tilley, T. D. J. Catal. 2002, 206, 49-59.

3. Sheldrick, G. M., SHELXT-2014/5. University of Götingen: Göttingen, Germany, 2014.

4. Sheldrick, G. Acta Crystallographica Section C 2015, 71, 3-8. 